\title{
Verletzung des Willkürverbots im Entscheid über die ordentliche Einbürgerung
}

Kommentar zum Urteil des Bundesgerichts 1D_1/2019 vom

18. Dezember 2019

Monika Plozza *

Das Bundesgericht hat sich erstmals zur Verletzung des Willkürverbots bei der Gewichtung der Integrationskriterien im Entscheid über die ordentliche Einbürgerung geäussert. Auch wenn sich das Bundesgericht mit dem altrechtlichen Verfahren befasst hat, ist der Entscheid dennoch bedeutend, da er das Willkürverbot genauer konturiert und für die aktuelle Einbürgerungspraxis Leitlinien vorgibt. Die vorliegende Urteilsbesprechung stellt die rechtlichen Grundlagen des Willkürverbots und der ordentlichen Einbürgerung dar, ordnet den Entscheid in den grösseren Kontext ein und analysiert dessen Bedeutung für die künftige Praxis von Einbürgerungsbehörden.

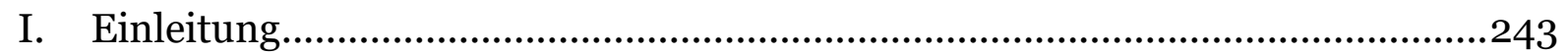

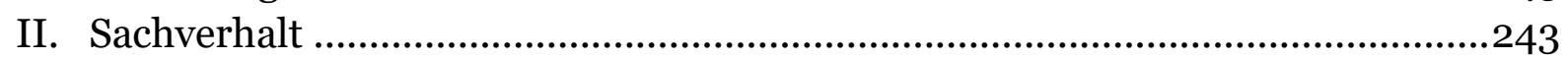

III. Erwägungen des Gerichts ................................................................................243

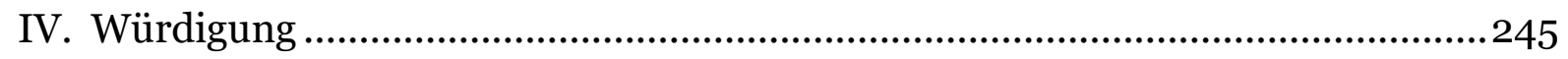

1. Das Willkürverbot (Art. 9 BV).....................................................................246

2. Die Praxis des Bundesgerichts zur Willkür in

Einbürgerungsangelegenheiten .................................................................246

3. Beurteilung der Integrationskriterien im Einbürgerungsverfahren ...............247

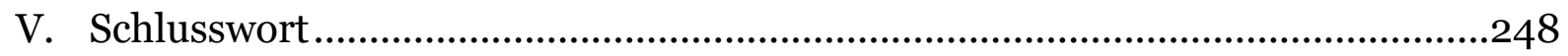

Zitiervorschlag: Monika Plozza, Verletzung des Willkürverbots im Entscheid über die ordentliche Einbürgerung, in: sui-generis 2020, S. 242

URL: $\quad$ sui-generis.ch/133

DOI: $\quad$ https://doi.org/10.21257/sg.133

* Monika Plozza (Monika.Plozza@unilu.ch), MLaw, ist wissenschaftliche Mitarbeiterin und Doktorandin an der Universität Luzern.

Dieses Werk ist lizenziert unter einer Creative Commons Namensnennung - Weitergabe unter gleichen Bedingungen 4.0 International Lizenz. 


\section{Einleitung}

1 Verletzungen des Willkürverbots in Einbürgerungsentscheiden beschäftigen das Bundesgericht schon seit mehreren Jahren. Beim vorliegenden Urteil handelt es sich somit nicht um eine Praxisänderung des Bundesgerichts. Diese erging bereits 2012, als das Bundesgericht seine Rechtsprechung mit BGE 138 I 305 änderte. Seither kann eine Willkürrüge in Einbürgerungsangelegenheiten mittels subsidiärer Verfassungsbeschwerde beim Bundesgericht vorgebracht werden. Das Bundesgericht hat seither verschiedentlich Urteile zu Verletzungen des Willkürverbotes in Einbürgerungsangelegenheiten gefällt. ${ }^{1}$ Interessant ist jedoch, dass sich das Bundesgericht erstmals zur Gewichtung der Integrationskriterien bei der ordentlichen Einbürgerung geäussert hat. Die vorliegende Urteilsbesprechung beschäftigt sich deshalb zunächst mit den rechtlichen Grundlagen des Willkürverbots und der ordentlichen Einbürgerung, ordnet den Entscheid in den grösseren Kontext ein und analysiert dessen Bedeutung für die künftige Praxis der Einbürgerungsbehörden.

\section{Sachverhalt}

2 Der Beschwerdeführer, ein italienischer Staatsangehöriger, wohnt mit seiner Ehefrau seit bald 30 Jahren in der Schwyzer Gemeinde Arth. Seine beiden Söhne kamen 1999 und 2006 in der Schweiz zur Welt. Seit 2001 führt der Beschwerdeführer einen eigenen Handwerksbetrieb. Am 20. März 2015 reichte er ein Gesuch um ordentliche Einbürgerung bei der Einbürgerungsbehörde in

\footnotetext{
Urteil des Bundesgerichts 1D_7/2017 vom 13. Juli 2018; Urteil des Bundesgerichts 1D_4/2018 vom 11. Juli 2019.
}

Arth ein. Diese wies 2017 das Einbürgerungsgesuch des Beschwerdeführers ab. Dagegen erhob er Beschwerde vor dem kantonalen Verwaltungsgericht. Die Beschwerde wurde 2018 abgewiesen. Mit subsidiärer Verfassungsbeschwerde gelangte der Beschwerdeführer an das Bundesgericht und beantragte, den negativen Entscheid des Einbürgerungsgesuchs aufzuheben und gutzuheissen.

3 Der Beschwerdeführer machte geltend, dass er die Voraussetzungen zur ordentlichen Einbürgerung erfülle. Er rügte insbesondere, dass die Entscheide der Einbürgerungsbehörde und des Verwaltungsgerichts das Willkürverbot von Art. $9 \mathrm{BV}^{2}$ verletzen. Das Verwaltungsgericht stützte sich, wie zuvor die Einbürgerungsbehörde der Gemeinde Arth, auf den Standpunkt, dass die Einbürgerung an seinen ungenügenden geographischen und kulturellen Kenntnissen sowie seiner ungenügenden gesellschaftlichen Eingliederung scheitere. Damit reiche es dem Gesuchsteller nicht zur ordentlichen Einbürgerung, obwohl die übrigen formellen und materiellen Voraussetzungen der Einbürgerung (unter anderem die übrigen Integrationskriterien) erfüllt seien.

\section{Erwägungen des Gerichts}

4 Da auf den Zeitpunkt der Gesuchseinreichung abgestellt wird, ist in diesem Fall noch die alte Rechtslage anwendbar (E. 2.1). Das Bundesgericht prüfte deshalb im vorliegenden Entscheid die Einbürgerungsvoraussetzungen nach dem aBüG3.

$2 \longdiv { \text { Bundesverfassung der Schweizerischen Eidge- } }$ nossenschaft vom 18. April 1999 (BV; SR 101).

3 Bundesgesetz vom 29. September 1952 über den Erwerb und Verlust des Schweizer Bürgerrechts (Bürgerrechtsgesetz, aB̈̈G; SR 141.o). 
Das Bundesgericht stellt zunächst die einschlägigen bundesrechtlichen Gesetzesartikel in E. 2.2 dar. Es führt aus, dass die formelle Voraussetzung der Wohnsitzpflicht nach Art. 15 aBüG im vorliegenden Fall nicht strittig sei. Weiter nennt es die materiellen Voraussetzungen zur Einbürgerung gemäss Art. 14 aBüG. Zudem führt das Gericht aus, dass die Kantone in der Ausgestaltung der Einbürgerungsvoraussetzungen einen gewissen Spielraum besitzen. Somit können die Kantone auch Konkretisierungen der bundesrechtlich verankerten Eignungskriterien vornehmen. In E. 2.3 stellt das Bundesgericht fest, dass der Kanton Schwyz gewisse Ausführungen zur «Eignung» vorgenommen hatte, jedoch die Anforderungen über den strittigen Punkt der «Lebensgewohnheiten, Sitten und Gebräuche» (Art. 14 lit. b aBüG) nicht weiter konkretisiert hatte.

6 Die Frage der Integration beurteilt sich nach Auffassung des Bundesgerichts «nach den gesamten Umständen des Einzelfalls» (E. 2.5). Das Gericht verdeutlicht, dass die Einbürgerungsbehörden beim Entscheid über die Einbürgerung zwar über einen Ermessensspielraum verfügen, dies jedoch nicht in einem Entschliessungsermessen münden soll (E. 2.6). Mit anderen Worten, dass einer Person, die alle gesetzlichen Einbürgerungsvoraussetzungen erfüllt und somit integriert ist, die Einbürgerung nicht verweigert werden darf. Andernfalls würde eine solche Praxis gegen das Willkürverbot von Art. 9 BV und das Rechtsgleichheitsgebot von Art. 8 Abs. 1 BV verstossen. Das Bundesgericht prüft deshalb, ob die Sachverhaltsfeststellungen der Vorinstanz das Willkürverbot verletzen (E. 3).
7 In E. 4.3 stellt das Bundesgericht fest, dass es sich bei den Integrationskriterien der «Eingliederung in die hiesigen Verhältnisse und das Vertrautsein mit den schweizerischen und lokalen Lebensumständen» um unbestimmte und deshalb auslegungsbedürftige Rechtsbegriffe handelt. Unter diese Kriterien fallen einerseits die wirtschaftliche und soziale Eingliederung und andererseits die Grundlagenkenntnisse der Staatskunde, Geschichte, Geografie sowie die kulturellen Sitten und Gebräuche. Dabei betont das Gericht, dass es bei den Einbürgerungsvoraussetzungen nicht um ein zu bestehendes Fachexamen gehen dürfe, sondern lediglich um eine Beurteilung von Lebenssachverhalten und Grundkenntnissen des Allgemeinwissens. Deshalb ist von Spitzfindigkeiten abzusehen. Des Weiteren soll die Messlatte nicht überhöht angesetzt werden. Von den Gesuchstellenden darf folglich nicht mehr verlangt werden, als von Schweizerinnen und Schweizern mit Wohnsitz in derselben Gemeinde erwartet werden kann.

8 Das Gericht erläutert in E. 4.4 die Anforderungen an eine ausgewogene Beurteilung der einzelnen Integrationskriterien. Obschon die kantonalen und kommunalen Behörden eine gewisse Gewichtung einzelner Gesichtspunkte vornehmen können, dürfen diese Bewertungen nicht in einem Missverhältnis zu den übrigen Kriterien stehen.

9 Die Vorinstanz war zum Schluss gekommen, dass die gesellschaftliche Integration des Beschwerdeführers den Anforderungen an die Einbürgerung nicht genüge. Dabei nahm sie Bezug auf Referenzauskünfte von vier Personen, welche dem Gesuchsteller ein durchschnittliches 
Ausmass an sozialen Kontakten mit Nachbarn und Gemeindeeinwohnern zusprachen. Die Vorinstanz führte jedoch nicht aus, weshalb die gesellschaftliche Eingliederung des Beschwerdeführers den Einbürgerungsanforderungen nicht genügte. Aus diesem Grund erwägt das Bundesgericht in E. 4.5.1, dass die Annahme, der Gesuchsteller habe keine Kontakte zur einheimischen Bevölkerung, jeglicher Lebenserfahrung widerspricht. Dies insbesondere deshalb, weil der Beschwerdeführer seit 2001 ein eigenes Gipsergeschäft führt und somit entgegen den Feststellungen der Vorinstanz aufgrund seiner selbstständigen Tätigkeit mit Sicherheit Kontakte zur einheimischen Bevölkerung pflegt.

Schliesslich stellte sich die Vorinstanz auf den Standpunkt, das Testergebnis der Prüfung über die geographischen und kulturellen Kenntnisse würde den Anforderungen zur Einbürgerung nicht genügen. Dazu führt das Bundesgericht in E. 4.5.3 aus, dass - obwohl der Beschwerdeführer bei den geographischen und kulturellen Kenntnissen keine Höchstleistung erbrachte - er doch die Hälfte der gefragten Sachverhalte ganz oder der Spur nach beantworten konnte. Ihm könne beispielsweise nicht vorgeworfen werden, dass er nicht wusste, dass im Tierpark Goldau Bären und Wölfe im gleichen Gehege leben oder dass ihm der Begriff «Ländler» nichts sagen würde. Hierzu führt das Bundesgericht aus, dass die Einbürgerungsbehörde auffallend spezifische Antworten vom Beschwerdeführer forderte. Dabei ist jedoch nicht wie in einem Fachexamen auf die erreichte Punktzahl abzustellen. Entscheidend sei vielmehr, ob er über Grundkenntnisse im fraglichen Lebens- bereich verfüge, selbst wenn sie keine Details kenne.

11 Das Bundesgericht stellt deshalb in E. 4.6 klar, dass der Beschwerdeführer, der seit 30 Jahren in der Schweiz lebt, trotz einem gewissen Vorbehalt bei den geographischen und kulturellen Kenntnissen dennoch die Einbürgerungsvoraussetzungen erfüllt. Das Gericht betont, dass ein geringer Mangel bei den geographischen und kulturellen Kenntnissen durch die übrigen erfüllten Einbürgerungskriterien ohne Zweifel aufgewogen wird. Die Verweigerung der Einbürgerung steht folglich in einem klaren Missverhältnis zur Gewichtung aller materiellen Einbürgerungsvoraussetzungen. Die vorgenommene Gewichtung der materiellen Einbürgerungsvoraussetzungen ist aus diesem Grund unhaltbar. Das Bundesgericht schliesst mit dem Fazit ab, dass der Entscheid der Vorinstanz das Willkürverbot von Art. 9 BV verletzt habe.

\section{Würdigung}

12 Willkürrügen zu Einbürgerungsentscheiden beschäftigen das Bundesgericht schon seit mehreren Jahren. Bemerkenswert ist am vorliegenden Urteil des Bundesgerichts, dass es sich erstmals zu einer Verletzung des Willkürverbots bei der Gewichtung der Integrationskriterien äussert. Somit stellt der vorliegende Entscheid ein Novum dar. Nachstehend wird zunächst auf das Willkürverbot (Art. 9 BV) eingegangen. Danach wird die Praxis des Bundesgerichts bei Willkürrügen in Einbürgerungsentscheiden mit Blick auf die früheren prozessualen Hürden präsentiert. Schliesslich wird die Bedeutung des vorliegenden Entscheids analysiert. 


\section{Das Willkürverbot (Art. 9 BV)}

13 Das Willkürverbot stellt nicht nur einen Grundsatz staatlichen Handelns, sondern auch ein selbstständiges verfassungsmässiges Recht dar.4 Die Funktion des Willkürverbots ist zweierlei: Einerseits nimmt es prozessual die Rolle als «letzten Rettungsanker» ein. Aus materieller Sicht stellt das Willkürverbot ein «Auffanggrundrecht» dar. Dies bedeutet, dass es erst dann zum Tragen kommt, sofern nicht auf ein anderes Grundrecht abgestellt werden kann.5

Wie soeben ausgeführt, bindet das Willkürverbot als Grundsatz staatlichen Handelns (Art. 35 Abs. 1 BV) alle Akteure bei der Wahrnehmung staatlicher Aufgaben (Art. 35 Abs. 2 BV). Adressaten des Willkürverbots sind sowohl der Gesetzgeber als auch die rechtsanwendenden Behörden. Für die kantonalen und kommunalen Behörden als Rechtsanwender ist das Willkürverbot in drei Konstellationen relevant: (1) bei der Auslegung von Rechtssätzen, (2) bei der Ausübung von Ermessen und (3) bei der Feststellung des Sachverhalts. ${ }^{6}$ Im vorliegenden Urteil hat das Bundesgericht einzig geprüft, ob «die Sachverhaltsfeststellungen der Vorinstanz an einem massgeblichen Mangel leiden oder in allgemeiner Weise willkürlich erscheinen» (E. 3). Im Rahmen der Sachverhaltsfeststellung kann Willkür vorliegen, wenn «(1) Sachverhaltsfeststellungen klar den Tatsachen widersprechen oder (2) Beweiswürdigungen im Lichte der Tatsachen nicht haltbar sind».7

Ulrich Häfelin/Walter Haller/Helen Keller/ Daniela Thurnherr, Schweizerisches Bundesstaatsrecht, 9. Aufl., 2016, Rz. 807.

5 Felix Uhlmann, Das Willkürverbot (Art. 9 BV), 2005, Rz. 337.

6 Regina Kiener/Walter Kälin/Judith Wyttenbach, Grundrechte, Bern 2018, S. $411 \mathrm{ff}$.

7 Kiener/Kälin/Wyttenbach (Fn. 6), S. 414 Rz. 26.
15 Schliesslich muss die Rechtsanwendung im Ergebnis als willkürlich erscheinen. In solchen Fällen stellt sich lediglich die Frage, ob eine willkürfreie Argumentation ein anderes Resultat hervorgebracht hätte. Die Willkür stellt deshalb nicht auf die Motive der handelnden Akteure ab. Mit anderen Worten hängt die Willkür einer Verfügung nicht von der abgegebenen Begründung ab. Somit liegt dem Willkürverbot eine objektive Komponente inne. ${ }^{8}$

\section{Die Praxis des Bundesgerichts bei Verletzungen des Willkürverbots in Einbürgerungsverfahren}

16 Insbesondere die ordentliche Einbürgerung galt als eines der letzten Gebiete, das sich in einem scheinbar «rechts- und gerichtsfreien Raum»9 bewegte. Obwohl Einbürgerungsverfahren lange Zeit als rein politischer Akt verstanden wurden, zeigt die Praxis des Bundesgerichts jedoch auf, dass diese Auffassung nicht haltbar ist. So erklärte das Bundesgericht etwa die Volksinitiative der Schweizerischen Volkspartei (SVP), nach der Einbürgerungen in der Stadt Zürich an der Urne entschieden werden sollten, für ungültig. ${ }^{10}$ Dieser Ansicht folgte es auch im Entscheid im Kontext der Urnenabstimmung über Einbürgerungsgesuche der Gemeinde Emmen und bejahte eine Verletzung sowohl der Begründungspflicht als auch des Diskriminierungsverbots. ${ }^{11}$ Das Bundesgericht erklärte, dass trotz fehlendem Rechtsanspruch auf Einbürgerung das Verfahren nicht in einem

8 Axel Tschentscher, in: Basler Kommentar, Bundesverfassung, 2015, N 3 zu Art. 9 BV; Christoph Rohner, in: St. Galler Kommentar, 3. Aufl., 2014, Rz. 7 zu Art. 9 BV.

9 Uhlmann (Fn. 5), Rz. 266.

10 BGE 129 I 232.

11 BGE 129 I 217. 
rechtsfreien Raum stattfinden dürfe. Dem Entscheid über die Einbürgerung liege eine einzelfallbezogene, d.h. individuell-konkrete Entscheidung zugrunde. Demzufolge erfüllt der Einbürgerungsentscheid auch die Merkmale einer Verfügung. Die Behörden hätten sich deshalb an die einschlägigen Verfahrensbestimmungen zu halten und seien an das Willkür- und Diskriminierungsverbot gebunden.12 In der Folge wurde die Begründungspflicht in das Bürgerrechtsgesetz (Art. 15b aB̈̈G) aufgenommen. ${ }^{13}$

Gemäss bundesgerichtlicher Rechtsprechung und herrschender Lehre besteht kein Rechtsanspruch auf Einbürgerung. Lange konnten sich Beschwerde führende Personen somit lediglich auf das Willkürverbot stützen, wenn ihnen eine Gesetzesbestimmung einen Rechtsanspruch einräumte. Deshalb blieb ihnen der Weg zum Bundesgericht in Einbürgerungsangelegenheiten verwehrt. Mit BGE 138 I 305 änderte das Bundesgericht jedoch seine Praxis. Dabei stellte es auf das revidierte BüG14 ab, das neu eine Begründungspflicht und Rechtsweggarantie für Einbürgerungen statuierte (Art. 15b bzw. Art. 50 aBüG).

18 Die Gesetzesänderung bewirkte, dass bei den betroffenen Personen die berechtigte Erwartung geweckt wurde, «einen willkür- und diskriminierungsfrei getroffenen Entscheid zu erhalten, welcher das Rechtsgleichheitsgebot beachtet».15 Mit Art. 14 aBüG bestand laut Bundesgericht

12 BGE 129 I 232 E. 3.3.

13 Martina Caroni/Nicole Scheiber/Christa Preisig/ Margarite Zoeteweij, Migrationsrecht, 4. Aufl., 2018, S. 544 ff.

14 Bundesgesetz vom 20. Juni 2014 über das Schweizer Bürgerrecht (Bürgerrechtsgesetz, BüG; SR 141.0).

15 BGE 138 I 305 E. 1.4.2. eine hinreichend klar umschriebene Rechtsposition, welche das Vorbringen der Willkürrüge vor Bundesgericht ermöglichte. Damit können Gesuchstellende, trotz fehlendem Rechtsanspruch auf Einbürgerung, eine Verletzung des Willkürverbots vor Bundesgericht rügen. Seither wurden wiederholt subsidiäre Verfassungsbeschwerden (Art. 115 BGG) wegen Verletzungen des Willkürverbots in Einbürgerungsverfahren erhoben. ${ }^{16}$

\section{Beurteilung der Integrationskrite- rien im Einbürgerungsverfahren}

19 Nach Ansicht der Lehre darf bei der Beurteilung, ob die Integrationskriterien erfüllt sind, nicht lediglich auf ein einzelnes Kriterium abgestellt werden. Erfüllt eine einbürgerungswillige Person eines der Kriterien nicht, darf die Einbürgerung nicht allein deswegen verweigert werden. Es ist vielmehr auf das Gesamtbild abzustellen. Ein unterdurchschnittliches Ergebnis in einem Bereich kann gleichwohl durch überdurchschnittliche Kenntnisse in anderen Punkten ausgeglichen werden. Ausnahmsweise kann jedoch ein Punkt für sich alleine den Ausschlag geben, wenn er besonders schwer wiegt, wie zum Beispiel eine erhebliche Straffälligkeit. ${ }^{17}$

20 Auch das Bundesgericht folgt der herrschenden Lehre und äusserte sich schon in früheren Urteilen zur Gewichtung der

$16 \overline{\text { Urteil des }}$ Bundesgerichts $1 \mathrm{D} \_7 / 2017$ vom 13. Juli 2018; Urteil des Bundesgerichts 1D_4/2018 vom 11. Juli 2019.

17 Laura Campisi, Die rechtliche Erfassung der Integration im schweizerischen Migrationsrecht: zwischen rechtlichen Vorgaben und innenpolitischen Realitäten, 2014, S. 247; Marc Spescha/ Antonia Kerland/Peter Bolzli, Handbuch zum Migrationsrecht, 2. Aufl., 2015, S. 415; Peter Uebersax, Der Begriff der Integration im schweizerischen Migrationsrecht, ASYL 4/o6, S. 9. 
Integrationskriterien. Dabei betonte es, dass eine Gesamtwürdigung vorgenommen werden muss. ${ }^{18}$ Ein Urteil ist in dieser Sache jedoch bis anhin nicht ergangen.

Da sich das vorliegende Urteil zum altrechtlichen Einbürgerungsverfahren äussert, ist hervorzuheben, dass die «Eignung» nach Art. 14 aB̈̈G mit der Integration gleichzusetzen ist. Dabei fällt nicht nur lit. a «in die schweizerischen Verhältnisse eingegliedert», sondern alle Voraussetzungen (lit. a-d) als Integrationskriterien in Betracht. ${ }^{19}$ Mit der Revision des BüG wurde der Begriff «Eignung» durch den Begriff der «Integration» ersetzt (Art. 11 und 12 BüG). Das BüG setzt mit Art. 11 lit. a eine erfolgreiche Integration voraus. Art. 12 BüG statuiert eine nicht abschliessende Liste von Integrationskriterien. Diese Kriterien stellen eine Verschärfung im Vergleich zur alten Version dar und bieten den Behörden auch heute noch einen relativ grossen Ermessensspielraum. ${ }^{20}$

Auch wenn sich der vorliegende Bundesgerichtsentscheid zum altrechtlichen Einbürgerungsverfahren äussert, ist er auch für Einbürgerungsgesuche, die ab dem 1. Januar 2018 eingereicht wurden, von grosser Bedeutung. Dies insbesondere, da in den letzten Jahrzehnten bei der Einbürgerung ein Paradigmenwechsel stattgefunden hat. Während früher die Annahme bestand, dass der Integrationsprozess erst mit der Einbürgerung

\footnotetext{
8 BGE 141 I 60.

19 Campisi (Fn. 17), S. 247.

20 Barbara von Rütte, Das neue Bürgerrechtsgesetz und dessen Umsetzung in den Kantonen, in: Alberto Achermann/Véronique Boillet/Martina Caroni/Astrid Epiney/Jörg Künzli/Peter Uebersax, (Hrsg.), Jahrbuch für Migrationsrecht /Annuaire du droit de la migration 2017-2018, 2018.
}

beginnen könne, wird heute die Integration als Bedingung für die Einbürgerung angesehen. Wie zuvor im aBüG erachtet das neue BüG die Integration ebenfalls als ein Grunderfordernis für die Einbürgerung und misst ihr zudem eine scheinbar noch grössere Wichtigkeit bei. ${ }^{21}$ Deshalb ist es umso bedeutender, dass die Einbürgerungsbehörden eine ausgewogene und willkürfreie Gewichtung der Integrationskriterien vornehmen.

\section{Schlusswort}

23 Das vorliegende Bundesgerichtsurteil konturiert erstmals das Willkürverbot bei der Bewertung der Integrationskriterien und gibt damit die Leitlinien zur Handhabung im Einbürgerungsverfahren vor. Auch die kantonalen und kommunalen Behörden sind an die Grundrechte gebunden. Die behördlichen Sachverhaltsfeststellungen und das ausgeübte Ermessen bei der Bewertung der Integrationskriterien haben somit objektiv und innerhalb der rechtsstaatlichen Schranken zu erfolgen - jenseits von politischen Anschauungen und eigenem Gutdünken. Auch die Einbürgerungsbehörden dürfen nicht willkürlich entscheiden. Ein negativer Einbürgerungsentscheid darf schliesslich nicht mit einem lediglich geringen Mangel eines einzelnen Integrationskriterium gerechtfertigt werden, vielmehr ist eine Gesamtschau nötig. Dies ist insbesondere mit Blick auf das neue BüG wichtig, das den Einbürgerungsbehörden einen grossen Ermessensspielraum bei der Bewertung der Integrationskriterien einräumt. Der vorliegende Bundesgerichtsentscheid ist deshalb für die Einbürgerungsbehörden wegweisend.

21 Campisi (Fn. 17), S. 274. 